We have developed and evaluated simple tools to facilitate MI based counselling for reducing high risk sexual behaviour.

Method Patients attending a drop-in STI services reception were offered 20-30 min of MI-based risk-reducing counselling. Out of 110 eligible patients (56 men and 54 women) who had had at least 4 sexual partners during the previous year, 72 patients aged 17-34 years (mean 24.8) participated. A structured manual was developed containing 14 questions, the first 5 of which were aimed at developing and amplifying the patient's motivation to reduce risky behaviour. We discussed with the patients their own perception of STI risks and the importance of not becoming infected, not infecting sexual partners and avoiding pregnancy. At the end of the session, patients were asked to assess their future commitment to changing risky sexual behaviour and a plan for accomplishing this change was drawn up. To ensure treatment fidelity, MI skill was assessed using the Motivational Interviewing Treatment Integrity Coding System (MITI 3.0).

Results At baseline, 4 of 32 men (12.5\%) and 6 of 40 women (15\%) had a genital CT infection. After intervention, the incidence of CT was $6 \%$ in men and $0 \%$ in women in the following 12 months. Of those CT-infected at start, none was re-infected within the next year. At baseline, men reported on average 8 (4-35) partners in the previous 12 months and women $6(4-17)$. On a scale from 0 to 10 men rated their STI-risk in the previous year as $5.5(1-10)$ and women as $6.3(2-10)$. The corresponding rating for pregnancy risk was $2.7(0-8.5)$ for men and $3.3(0-9)$ for women. Men rated the importance of not becoming infected as $8.5(5-10)$ and women as 9.5 (5-10). The importance of not infecting a sexual partner was rated as $9.2(2.5-10)$ in men and $9.6(6-10)$ in women. The importance of avoiding pregnancy was rated as $9.2(5-10)$ in men and $9.5(2-10)$ in women. The ability to reduce sexual risks was judged as $8.4(5-10)$ in men and $8.6(3.5-10)$ in women. At the end of the session, patients' commitment to reducing STI risks was 9.1 (6-10) for men and 9.3 (8-10) for women.

Conclusion Brief MI-based single-session counselling aimed at assessing personal risks, resolving ambivalence, developing riskreduction strategies and implementing safer sex behaviours is effective in the reduction of high-risk sexual practices and CT incidence.

\section{P2-S4.05 ACCEPTABILITY OF MEDICAL MALE CIRCUMCISION AMONG MEN ATTENDING AN HIV VOLUNTARY COUNSELLING AND TESTING CENTER IN MYSORE, INDIA}

doi:10.1136/sextrans-2011-050108.337

${ }^{1} \mathrm{P}$ Madhivanan, ${ }^{1} \mathrm{~K}$ Krupp, ${ }^{1} \mathrm{~V}$ Srinivas, ${ }^{2} \mathrm{~J}$ Klausner. ${ }^{1}$ Public Health Research Institute of India, Mysore, India; ${ }^{2}$ University of California, , San Francisco, USA

Background There is currently limited information on the acceptability of medical male circumcision (MMC) in India. This study investigated the acceptability of MMC as a HIV prevention strategy among attendees of an HIV voluntary counselling and testing center (VCT) in Mysore, India.

Methods A cross-sectional survey was conducted among a consecutive sample of 300 men attending the VCT in Mysore, India, between April and August 2009. After obtaining the informed consent in Kannada, an interviewer administered survey collected demographic, sexual risk behaviour, perceived risk and acceptability of MMC information.

Results Most participants were Hindus (98.2\%); 1.5\% were Muslims, and $0.3 \%$ belonged to other religions. Fifty-three per cent of respondents had less than 7 years of schooling, $27.5 \%$ had $8-10$ years, $3.0 \%$ had attended high school and $16.0 \%$ had more than 12 years. Of participants, $71 \%$ were married and $45 \%$ made less than 4000 INR per month. About 244(73.9\%) said that they had heard of MMC. On being informed about the risks and benefits of male circumcision, $97.9 \%$ said that other men should consider MMC to reduce the risk of contracting HIV/STDs. When asked if they would consider being circumcised safely in a hospital or clinic with little/no pain and at low/no cost, only a third (32.1\%) said they would consider circumcision. The most important barriers to MMC reported by participants included MMC not being their cultural tradition (90\%), fear of stigma and rejection by friends and family (74.8\%) and pain (76.7\%).

Conclusions While MMC has been shown to be protective against heterosexual acquisition of HIV, little is known about whether it could be implemented in this primarily non-circumcising country. While this study found relatively low acceptability for MMC among VCT clinic attendees, more research on MMC acceptability among other populations, particularly those at high risk for HIV, is warranted.

\section{P2-S4.06 KNOWLEDGE OF HSV-2 SEROSTATUS IN ASYMPTOMATIC ADULTS MAY RESULT IN CHANGE IN SEXUAL BEHAVIOUR AND POSSIBLE RISK REDUCTION}

doi:10.1136/sextrans-2011-050108.338

M G Cuyugan, W Mulugeta, M Thabolingam-Haridas, T Wilson, M Augenbraun, T Elsassy, B Cerniglia. SUNY Downstate Medical Center, Brooklyn, USA

Background HSV-2 is the main cause of genital ulcer disease worldwide. It is frequently under- recognised because it is often clinically silent. Serologic testing for HSV antibody reliably identifies individuals infected with HSV-2. There is little literature investigating whether serologic screening of asymptomatic patients changes sexual behaviour and risk reduction.

Methods Patients $>18$ yrs old who came to an urban STD clinic in Brooklyn with no previous history of HSV or HIV, no genital ulcer at initial visit, and sexually active in the preceding 3-6 months were administered a questionnaire. HSV1/2 IgG testing using HerpesSelect (Becton Dickinson and Company in Sparks MD) was used and patients were told about the results after 1 week. Participants were educated regarding natural history of HSV and asked to come back after 3 months to complete a follow-up questionnaire. Changes in sexual behaviour including number of sexual partner, condom use and psychological stress were the primary outcome of interest of this study.

Results A total of 193 eligible participants were included for analysis and follow-up is available for 101(51\%). There was no significant difference among baseline characteristics (age, gender, no. of sexual partners) among participants. Majority of participants were heterosexuals (95.8\%) and black immigrants (92.3\%). The seroprevalence of HSV2 IgG was 35\%. More women were HSV + (67\%) and more HSV + participants reported having non-steady partners ( $p$ 0.027). When analysing change (from baseline to follow-up), there was a significant decrease in number of non-steady partners among HSV + and HSV-participants (p 0.004 and p 0.0049 respectively). There was no statistically significant difference in the frequency of condom use between HSV + and HSV - participants ( $p=0.448$ and $p=0.91$ ). Discussion: There was a significant decrease in the number of non-steady partners after knowing HSV status among both HSV + and HSV-participants but no change in frequency of condom use. This finding is important because this suggests that knowledge of HSV status may impact sexual behaviour that can potentially reduce risk of transmission. However, both groups had a significant change in the number of non-steady partners regardless of HSV results, which may suggest that being tested for HSV-2 is enough to alter certain risky sexual behaviours. This can be potentially useful to patients visiting STD clinics for screening. 